\title{
Autour de Holger Meins. Documentaire et lutte armée dans l'entourage de la DFFB après 1969
}

In Sachen Holger Meins. Dokumentarfilm und bewaffneter Kampf im Umfeld der Deutschen Film- und Fernsehakademie Berlin

About Holger Meins : Documentary and Armed Struggle within the DFFB after 1969

Jeremy Hamers

\section{OpenEdition}

Journals

Édition électronique

URL : https://journals.openedition.org/ceg/8130

DOI : $10.4000 /$ ceg. 8130

ISSN : 2605-8359

\section{Éditeur}

Presses Universitaires de Provence

Édition imprimée

Date de publication : 1 mai 2013

Pagination : 113-129

ISBN : 0751-4239

ISSN : 0751-4239

\section{Référence électronique}

Jeremy Hamers, « Autour de Holger Meins. Documentaire et lutte armée dans l'entourage de la DFFB après 1969 », Cahiers d'Études Germaniques [En ligne], 64 | 2013, mis en ligne le 02 janvier 2020, consulté le 02 juin 2021. URL : http://journals.openedition.org/ceg/8130 ; DOI : https://doi.org/ $10.4000 /$ ceg. 8130 


\title{
Autour de Holger Meins. Documentaire et lutte armée dans l'entourage de la DFFB après 1969
}

\author{
Jeremy HAMERS \\ Université de Liège
}

\section{Introduction}

Créée dans la foulée du renouveau du cinéma allemand de 1962 (Manifeste d'Oberhausen), la Deutsche Film- und Fernsehakademie Berlin (DFFB) ouvre ses portes à Berlin-ouest en 1966. Dans un contexte politique qui enfantera notamment les manifestations berlinoises contre la venue du Shah un an plus tard, les premiers étudiants en cinéma, en collaboration avec leurs condisciples de la Freie Universität et de la Technische Universität, se retrouvent rapidement aux commandes d'actions, de happenings, de réunions, de tribunaux symboliques, etc. C'est l'époque des ciné-tracts, fonctionnels, bruts, mis au service de la seule lutte marxiste' ${ }^{1}$ La collaboration entre les universitaires, l'opposition extraparlementaire et les apprentis cinéastes est à ce point évidente qu'elle a fondamentalement déterminé de nombreux travaux critiques et scientifiques relatifs aux films réalisés à la DFFB entre 1966 et 1969. La plupart de ces travaux sur la production des premières années de la DFFB analysent en effet les pamphlets filmés et autres ciné-tracts à la seule lumière de la lutte étudiante qui, rapidement, subit le détachement de certains radicaux qui s'en iront fonder la Rote Armee Fraktion (RAF, Fraction Armée Rouge), la Bewegung 2. Juni (Mouvement du 2 juin) et d'autres groupes armés, seul moyen pour ces nouveaux guérilleros de préserver l'authenticité

1 «Car le travail cinćmatographique socialiste doit être partic du travail politiquc. Et lc travail cinćmatographique socialiste ne peut être réalisé sans la praxis du travail politique», Holger MEINS, Michacl LUKASIK, Günter-Pcter STRASCHEK, «Schülerfilmprojekt» (1968), in Petra KRAUS, Natalic LETENEWITSCH, Ursula SAEKEL et al., 1977-1997. Deutschland im Herbst. Terrorismus im Film, Münehen, Schriftenreihe Münchner Filmzentrum, 1997, p. 96. 
d'une lutte qui se voyait progressivement désamorcée par son institutionnalisation et son passage à la culture reconnue ${ }^{2}$.

Évidemment, l'histoire se prête particulièrement bien à ce déterminisme politico-judiciaire, étant donné que la première année de la DFFB compte en son sein deux futurs terroristes, en les personnes de Holger Meins (RAF) et de Philip Werner Sauber (Bewegung 2. Juni) et que certains de leurs films réalisent effectivement la subordination totale du cinéma à l'action directe ${ }^{3}$. Il serait néanmoins réducteur de considérer tous les premiers films politiques de la DFFB comme de simples signes avant-coureurs d'une radicalisation de la lutte et d'un passage à la violence armée. Rappelons en effet qu'au moment où les agit-films et ciné-tracts produits avec les moyens techniques de la DFFB circulent de teach-ins en manifestations politiques, Harun Farocki et Helke Sander notamment réalisent leurs premiers films réflexifs qui mettent déjà la lutte à distance ou, plus exactement, qui subordonnent la lutte politique à une réflexion en profondeur sur le pouvoir du cinéma et le lieu d'où parle et filme le réalisateur. Citons parmi d'autres les court-métrages Die Worte des Vorsitzenden (Les paroles du Président, H. Farocki, ass. réal. H. Sander, 1967), Ihre Zeitungen (Leurs journaux, H. Farocki, ass. réal. H. Sander, 1968), ou encore Unlöschbares Feuer (Feu inextinguible, H. Farocki, ass. réal. H. Sander, 1969), qui se font les miroirs ironiques et réflexifs de la lutte par le cinéma bien plus qu'arme au poing, engageant le corps et la figure du cinéaste comme révélateur des limites d'un cinéma engagé, une «surface témoin » qui agit comme une échappatoire possible à l'usage médiatique du choc, de la pitié et de l'outrance ${ }^{4}$.

Passée l'époque des enthousiasmes ludiques et réflexifs, le cinéma allemand est confronté dès le début des années soixante-dix à l'effritement d'une partie de la gauche extraparlementaire ainsi qu'à l'avènement du terrorisme d'extrême gauche. Dans l'intervalle, certains apprentis cinéastes

2 Au sujet de l'institutionnalisation de la lutte, voir notamment: Dominique LINHARDT, «L'État et ses épreuves : éléments d'une sociologie des agencements étatiques », Papiers de Recherche du CSI, $\mathrm{n}^{\circ}$ 9, 2008. url : www.csi.ensmp.fr; Jeremy HAMERS : « Notes sur quelques provocations politiques (Kommune I/ Yes Men / Leo Bassi) », Prospero European Review. Theatre and Research, $\mathrm{n}^{\circ}$, 2011, url : http:/www.t-n-b.fr/en/prospero/europeanreview/fiche.php?id=70\&edition=9\&lang $=2$.

3 On ne compte plus les textes et travaux qui font par exemple référence au célèbre Herstellung eines Molotow Cocktails, attribué à Holger Meins et considéré comme perdu, un agit-film devenu emblème d'une époque de la DFFB.

4 Dans son analyse de Unlöschbares Feuer, Tilman Baumgärtel souligne de façon tout à fait judicieuse que le geste automutilant d'un réalisateur s'écrasant une cigarette sur l'avant-bras «parce qu'il ne peut que donner une faible image de l'effet du Napalm », scelle un passage du militant à l'auteur, du cinéma fonctionnel à un cinéma réflexif. Par ailleurs, Baumgärtel suggère aussi que Die Worte des Vorsitzenden, notamment, constitue, quant à lui, un plaidoyer discret pour le passage à la lutte armée. Nous ne le suivons pas dans cette interprétation, car il nous semble que la distanciation suggérée par ce film exprime déjà un premier détachement de la lutte primaire et directe. Tilman BAUMGÄRTEL, « Bildnis des Künstlers als junger Mann. Kulturrevolution, Situationismus und Focus-Theorie in den Studentenfilmen von Harun Farocki », in Rolf AURICH, Ulrich KRIEST, Der Ärger mit den Bildern. Die Filme von Harun Farocki, Konstanz, UVK Medien, 1998, p. 155-178. 
ont fait le choix de la lutte armée clandestine, tandis que, entre remords et soulagement d'avoir fait le " bon » choix, d'autres font le dur apprentissage de l'autonomie financière, une expérience qui, pour certains, devient vite incompatible avec l'engagement de la première heure. La symétrie semble évidente : si pour Holger Meins par exemple, le choix de la lutte armée s'est fait au détriment de la pratique cinématographique ${ }^{5}$, pour les jeunes réalisateurs, l'option cinématographique leur impose un renoncement politique partiel. En somme, cinéma et lutte semblent, in fine, incompatibles ${ }^{6}$. Et l'histoire retiendra que, dès 1969 , les réalisateurs issus de la première promotion de la DFFB s'éloignent du militantisme.

Cet apparent apaisement du cinéma militant berlinois est encouragé dès 1972 par une censure indirecte qui frappe tout réalisateur qui manifesterait un intérêt, aussi circonstancié soit-il, pour le phénomène de la lutte armée. Dans le climat de chasse aux sorcières qui règne alors en Allemagne et qui expose à l'opprobre médiatique tout comme aux poursuites judiciaires tout intellectuel et artiste qui s'aventurerait sur le terrain houleux d'une compréhension, même relative, de la démarche terroriste, les principaux financeurs, à savoir les commissions publiques et les chaînes de télévision, se montrent en effet particulièrement frileux vis à vis de tout projet qui se rapprocherait ou questionnerait de façon un peu trop indépendante la question terroriste allemande. Un autre problème entrave par ailleurs le travail spécifique des documentaristes : les actes terroristes, leurs principaux acteurs, tout comme la répression et les actes de prévention qui lui font face, restent largement inaccessibles et « infilmables ». Soit ils sont, par définition, secrets et consommables seulement par le biais des ruines qu'ils laissent derrière eux, soit ils sont nécessairement marqués du sceau de la mise en scène médiatique (par la police comme par les terroristes) et de la violence sémantique ${ }^{7}$.

5 Se souvenant de son condisciple Holger Meins dans un entretien avec Tilman Baumgärtel, Harun Farocki confirme l'existence d'une césure effective entre pratique cinématographique et engagement dans la lutte armée, que l'auteur déduit aussi de son propre parcours : " Er dachte das bestimmt zusammen, Ästhetik und Politik. Ich glaube, daß er unheimlich gespalten war, ob er Filme oder Politik machen sollte. [...] Retrospektiv weiß ich, daß ich nie in den Untergrund gegangen wäre, weil ich mich viel zu sehr darauf festgelegt hatte Filme zu machen oder intellektuell-künstlerisch tätig zu sein. " Harun FAROCKI, Tilman BAUMGÄRTEL, "'Holger dachte Ästhetik und Politik zusammen'. Gespräch mit Harun Farocki über den Filmstudenten Holger Meins und seinen Weg in die RAF ». Cet entretien est consultable en ligne sur le site de Tilman Baumgärtel : url: www.thing.de/tilman/ holgermeins.htm.

6 Concernant le passage d'un engagement politique direct à un travail plus formel et réflexif, voir aussi: Frank ARNOLD, "Die Spannung zwischen gesellschaftspolitischem Denken und künstlerischem Talent. Ein Gespräch mit den ehemaligen Studienleitern Hans Helmut Prinzler und Karl Saurer ", in Frank ARNOLD, Deutsche Film- und Fernsehakademie Berlin (dffb). Eine Retrospektive 1966-1986, Oberhausen, Westdeutsche Kurzfilmtage Oberhausen, 1989, p. 7-14.

7 Concernant les rapports d'interdépendance entre terrorisme d'extrême-gauche allemand et médias de masse, et les enjeux dramatiques des ruines sous forme de décors vides, voir notamment : Jeremy HAMERS, «Un théâtre sans acteurs. L'enlèvement de Hans-Martin Schleyer par la Fraction Armée Rouge », Cahiers Louis-Lumière, nº 6, 2009, p. 53-67. 
Selon la plupart des historiens qui se sont penchés sur cette période, l'émergence de la lutte armée provoque donc une césure, un écart infranchissable entre les étudiants devenus combattants et ceux d'entre eux qui ont choisi le septième $\operatorname{art}^{8}$. À en croire la plupart des filmographies parues sur le sujet, ce divorce entre lutte directe et cinéma s'accompagne d'un désintérêt forcé du cinéma documentaire pour la lutte armée ${ }^{9}$. Si dans ces panoramas les fictions ne manquent pas, l'histoire ne retient pour ainsi dire aucun documentaire indépendant qui s'intéresserait au fait terroriste après $1969^{10}$. Et ce silence documentaire ne trouverait un terme qu'en 1977/1978 avec la sortie du film collectif Deutschland im Herbst (L'Allemagne en automne, Alexander Kluge, Volker Schlöndorff, Rainer Werner Fassbinder et al.) qui ressusciterait un documentarisme critique et indépendant préoccupé par le terrorisme d'extrême-gauche allemand. Entre Herstellung eines Molotow Cocktails (Fabrication d'un cocktail molotov) attribué à Holger Meins (1967) ou Unlöschbares Feuer, et le célèbre film collectif de 1978, on assisterait donc à un temps mort documentaire. Pourtant, et notamment sous l'impulsion de divers groupes de vidéo-activistes mais aussi d'étudiants de la DFFB, plusieurs documentaires consacrés à des membres de la RAF ou de la Bewegung 2. Juni voient le jour dans cet intervalle. Produits au sein de structures a priori moins soumises au Diktat financier étatique, ces films n'ont, à ce jour, pas encore fait l'objet d'un recensement exhaustif et d'études approfondies. Dans la suite de ce texte, nous aimerions leur offrir un premier fondement encore partiel, en nous intéressant à deux de ces films qui démontrent qu'entre la militance directe de la seconde moitié des années soixante et l'éclairage apporté par le Nouveau Cinéma Allemand sur les événements de "l'automne allemand », Berlin n'a cessé d'inspirer un « contre-cinéma » doublement fondé sur la critique politique et la réflexion formelle.

Es stirbt allerdings ein Jeder, Frage ist nur wie und wie du gelebt hast (Tout le monde doit effectivement mourir un jour. La question est : comment et comment as-tu vécu. ${ }^{11}$ ) de Renate Sami (1975-1976) et Über Holger Meins (Au sujet de Holger Meins) de Gerd Conradt et Hartmut Jahn (1975-1982,

8 Voir notamment: Thomas ELSAESSER, « Harun Farocki : Filmmaker, Artist, Media Theorist 》, in Thomas ELSAESSER (éd.), Harun Farocki. Working on the Sightlines, Amsterdam, Amsterdam University Press, 2004, p. 17.

9 Il faut toutefois mentionner la filmographie établie par Anna Pfitzenmaier pour le périodique en ligne Zeitgeschichte-Online, qui déroge à cette constatation en référençant notamment quelques films dont il sera question ici et réalisés dans le giron de la DFFB au cours des années $70:$ http://www.zeitgeschichte-online.de/site/40208743/default.aspx

10 Selon Jan Dawson, l'autocensure des réalisateurs aboutit à la réalisation de plusieurs films de fiction qui préconisent, contre la problématisation explicite de certains événements, le traitement de cas particuliers et microcosmiques. Pour l'analyste, la fiction devient alors le lieu d'interrogations politiques qui se réfugient derrière l'alibi du cas particulier et fictif. Jan DAWSON, «The Sacred Terror », Sight and Sound, n'198, vol. 48/4 1979, p. 243.

11 Tout le monde doit effectivement mourir un jour. La question est : comment et comment as-tu vécu. Ce titre est un extrait de la dernière lettre de Holger Meins, écrite en prison, peu de temps avant son décès. 
analysé ici dans sa version inédite et inachevée de 1978) se focalisent tous deux sur le sort d'une figure absente, l'étudiant en cinéma Holger Meins devenu «terroriste » (RAF). Proposant une nouvelle approche des rapports problématiques entre militance et création, et prolongeant le travail réflexif initié par Farocki et Sander à la fin des années soixante, ces deux documentaires abordent le sort d'un combattant armé en conservant une liberté de ton et en résistant à la violence sémantique exercée par les médias à l'encontre des membres de la RAF. Leur singularité réside notamment, nous tenterons de le démontrer, dans leur usage de la figure de l'auteur, une figure qui est fondamentalement fragilisée et remise en question dans le contexte politique et artistique des années soixante-dix.

\section{Film à plusieurs voix}

Es stirbt allerdings ein Jeder. Frage ist nur wie und wie du gelebt hast, apparaît au premier visionnage comme un film d'entretiens qui entend rétablir « la vérité » au sujet d'un terroriste, Holger Meins, décédé deux ans plus tôt des suites d'une grève de la faim à la prison de Wittlich. Composé d'une succession d'entretiens avec différents proches, collègues et amis du terroriste décédé, Es stirbt allerdings ein Jeder ménage par ailleurs une place centrale à la réalisatrice. Elle y est non seulement visible et identifiable en tant qu'auteure qui tend le micro aux interviewés, mais elle y apparaît en outre comme une instance qui suscite et dirige les réponses de ses interlocuteurs en fonction de sa sensibilité et de sa curiosité propres. Cette place centrale et tout à fait exposée est déjà manifeste dans le générique. Ensuite, dès les premiers plans du film, on la retrouve, micro au poing, assise aux côtés de ses interlocuteurs. Dans le contexte de chasse aux sorcières médiatique des années soixante-dix, cette exposition ne relève toutefois aucunement d'un acte suicidaire. Arrêtée et incarcérée en détention préventive durant un an en 1970 parce qu'elle était suspectée d'avoir participé à un attentat contre le consulat américain (Amerikahaus) de Berlin, se révélant ensuite en 1973 dans un ouvrage collectif au titre éloquent de Wie man gegen Polizei und Justiz die Nerven behält ${ }^{12}$ (Comment garder les nerfs face à la police et à la justice), Sami n'est pas une activiste débutante dont l'anonymat constituerait une condition essentielle à la poursuite de ses activités ${ }^{13}$.

Par ailleurs, le film de Sami n'est pas réductible non plus à l'œuvre d'une réalisatrice dont le camp est établi depuis longtemps et dont la production ne

12 Klaus ESCHEN, Sybille PLOGSTEDT, Renate SAMI et al, Wie man gegen Polizei und Justiz die Nerven behält, Berlin, Rotbuch Verlag, 1973.

13 Selon la réalisatrice, c'est d'ailleurs le dédommagement financier perçu pour son acquittement qui lui permettra de réaliser son film sans apport financier extérieur. Heike BEHREND, Renate SAMI, "Holger Meins. Filmstudent von 1966 bis 1968 ", in Werner PETERMANN, Ralph THOMS (éd.), Kino-Fronten. 20 Jahre '68 und das Kino, München, Trickster Verlag, 1988, p. 19. 
se limiterait alors qu'à la réaffirmation d'un point de vue, d'un discours, d'une version des faits. Si Sami est la première réalisatrice et intervieweuse dans le film, elle y côtoie aussi plusieurs autres auteurs qui jouissent d'un statut comparable à l'intérieur de la structure pragmatique du film : celui d'une personne dont le souvenir subjectif constitue l'unique source d'information. Plusieurs passages dans le documentaire témoignent de cette démultiplication des auteurs. À plusieurs reprises, les vis-à-vis de Sami, pour la plupart des anciens étudiants de la DFFB devenus réalisateurs entre-temps, s'extraient de leur rôle de témoin pour devenir à leur tour les commentateurs de leurs propos, révélant ainsi le dispositif au sein duquel ils sont amenés à témoigner. Le cadre figé de l'interview est ainsi débarrassé, le temps d'un commentaire, de son carcan monodirectionnel.

Il en va ainsi lorsque le réalisateur, critique et ancien étudiant de la DFFB Hartmut Bitomsky se souvient du tournage de son film d'étude Vögelchen (Petit oiseau), cadré par Holger Meins. Lors de l'évocation de ses débuts en tant que réalisateur, Bitomsky fait directement référence au tournage de Sami, c'est-à-dire à la situation énonciative dans laquelle il se trouve lors de l'interview, cadré cette fois par Gerd Conradt : «Et Gerd, qui se trouve maintenant derrière la caméra, a tenu le rôle principal. Donc, c'était exactement l'inverse ${ }^{14}$.»

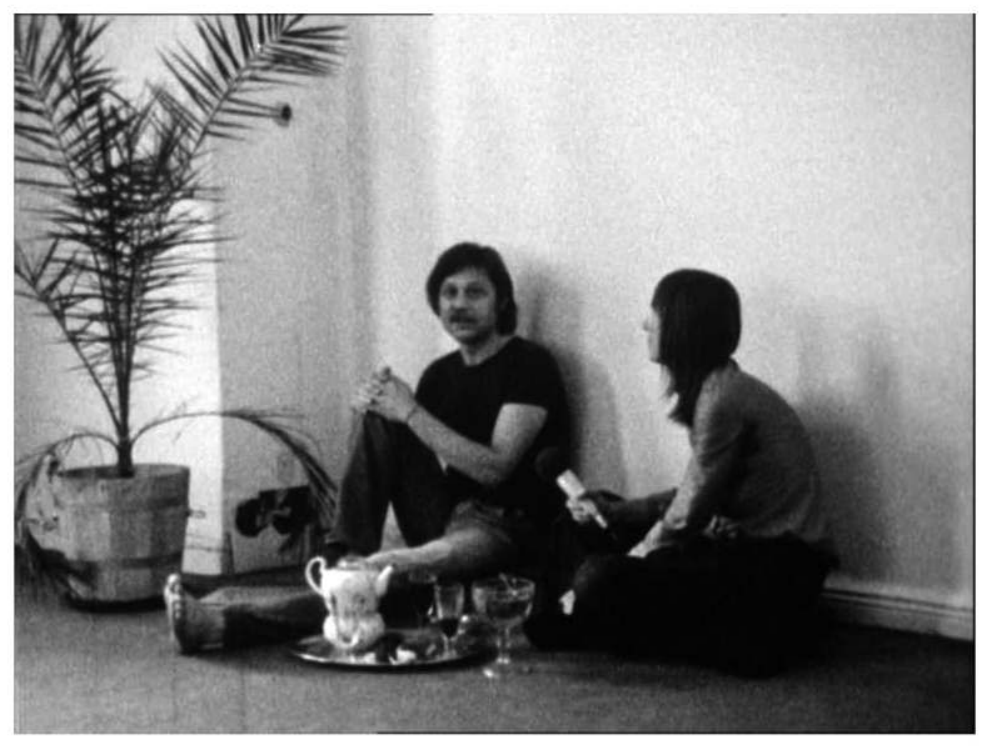

La mémoire-vidéo - dispositif et protagonistes 1 : Hartmut Bitomsky, Renate Sami. Es stirbt allerdings ein Jeder de Renate Sami (1975). Photogramme.

14 «Und der Gerd, der jetzt hinter der Kamera ist, hat die Hauptrolle gespielt, also genau umgekehrt. » Extrait des dialogues de Es stirbt allerdings ein Jeder. 
Sami autorise donc un démembrement partiel du dispositif de l'entretien classique qui permet à d'autres intervenants de prendre provisoirement la direction de l'entretien. Ce changement de rôle, indique que, même passagère, une prolifération des auteurs est possible. Cette prolifération abolit la frontière entre l'interviewer et l'interviewé, entre le détenteur de la parole et son vis-à-vis, définissant d'ordinaire le cadre canonique de l'entretien, et transforme donc le film de témoignages critiques en une ouvre à plusieurs voix. Cet éclatement du carcan monodirectionnel de l'interview classique ouvre également la voie à un cinéma documentaire engagé plus réflexif, grâce auquel le souvenir subjectif d'un ou plusieurs auteurs ne prend plus le contrepied simple de différents discours officiels (médias, police, gouvernement, etc.), mais se retourne littéralement sur lui-même, comme lorsque Hartmut Bitomsky se souvient de son engagement politique :

\begin{abstract}
- Renate Sami: In dem Wunsch also Filme anders zu machen als damals üblich oder, ihr habt damals ein Flugblatt unterschrieben „Film als Mittel zur Umgestaltung der Gesellschaft einzusetzen", seid ihr euch doch irgendwie einig gewesen. Der Holger hat das dann versucht also praktisch, politisch zu machen, und du bist beim Film geblieben. Wie sieht es eigentlich heute aus, wenn man Filme anders machen will? Also so konkrete Erfahrungen, die du beim Film oder beim Fernsehen gemacht hast. Könntest du darüber was erzählen?

- Hartmut Bitomsky: Damals haben wir uns halt von unserem Leben und unseren Möglichkeiten wahrscheinlich sehr viel mehr versprochen als das, was uns zugestanden wird. Die Zensur ist unheimlich stark. Ich meine nicht die Zensur in dem Begriff, sondern halt die Behinderung, die Hemmung von Arbeit, von Ideen und Fantasie, von sozialer Fantasie. Das ist so alles viel schlimmer als man von damals es gedacht hat. [...] In dem Moment wo die Sachen eine richtige gesellschaftliche, relevante Existenz haben, schauen einem auch sehr viele Leute auf die Finger. Und ganz schnell versiegt das Geld, versickert, verschwindet, kriegt man nichts mehr, wird abgenabelt. Insofern ist vieles, was wir uns damals gedacht haben, glaub ich doch sehr zu kurz gegriffen. Dann glaube ich also, dass in diesem Zeitpunkt, also zu dieser Zeit halt, die politischen Organisationen mit Film nicht viel anfangen können, weil Film so unendlich teuer ist, und dass man das Geld was da vielleicht in andere Sachen reinstecken müsste und nicht in Film ${ }^{15}$.
\end{abstract}

Comme l'indique cet échange, la résignation et la déception de Bitomsky figurent l'origine et les raisons du choix politique du personnage principal et absent, permettant aussi de dépasser une première fois l'inaccessibilité des événements. Holger Meins, grand absent du film, le terroriste dont on ne dispose plus que de représentations médiatiques et policières après son passage à la clandestinité, reçoit un visage par un effet de substitution. Le combattant armé est approché par son « contraire », le cinéaste qui n'a pas choisi la voie de la radicalité. Cette substitution ou représentation par procuration n'est pas sans rappeler le glissement déjà évoqué pour Unlöschbares Feuer de Harun Farocki : en ce qu'il sous-entend bien évidemment un renoncement à toutes les traces directes laissées par le

15 Renate SAMI, Hartmut BITOMSKY, dans Es stirbt allerdings ein Jeder. 
protagoniste principal (textes, manifestes, portraits, extraits de films, tableaux, etc.), le portrait par substitution présuppose un refus des images médiatiques dans ce cas - du terroriste et de la lutte armée en général.

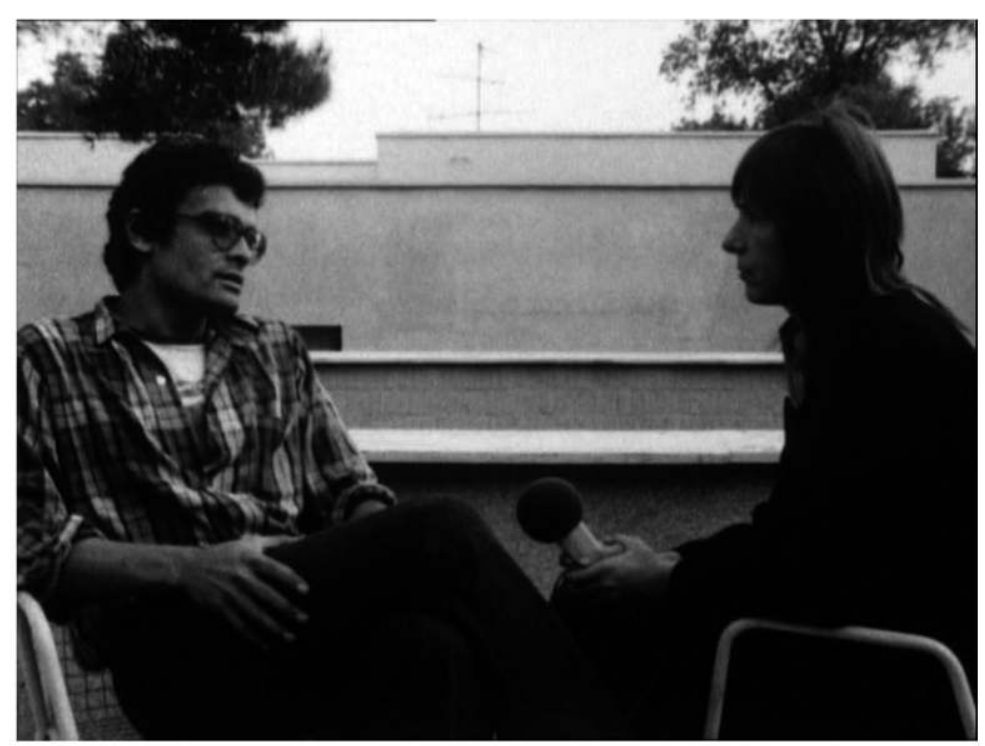

La mémoire-vidéo - dispositif et protagonistes 2 : Harun Farocki, Renate Sami. Es stirbt allerdings ein Jeder de Renate Sami (1975). Photogramme.

Ce refus de la représentation directe réapparaît à la fin du film, cette fois sous la forme d'une extinction de l'image, au moment précis d'un témoignage qui relate les dernières heures de Meins avant son passage à la clandestinité : 


\begin{abstract}
Und irgendwie war von dem Zeitpunkt an klar, dass der Holger einen anderen Weg gehen würde, hat das ganz allmählich, aber sehr bewusst vorbereitet und hat sich eigentlich richtig so Stück für Stück verabschiedet. Wir haben darüber kaum geredet, aber es war klar, dass er Sachen machen wollte, die in der Grunewaldstraße, die der Polizei bekannt war und wo auch viele wohnten und Kinder waren, dass er da nicht mehr leben konnte mit der Perspektive, die er für die Zukunft hatte. Ich erinnere mich noch als wir uns so allmählich darüber im Klaren wurden, dass wir nicht weiter zusammen leben würden, wie der Holger zum letzten Mal da war. Ich hab, glaub ich, wie meistens irgendwas genäht bei uns in der Küche. Und der Holger hat sich abgemeldet und er kam noch zum Schluss um richtig so auf Wiedersehen zu sagen. Mir war klar, dass ich ihn also wahrscheinlich nur in der Zeitung wieder sehen würde und so wahrscheinlich nicht mehr. Dann kamen, später dann, zwei Jahre später, die Bilder von seiner Verhaftung. Na ja, das andere ist ja klar, wie das dann gelaufen ist ${ }^{16}$.
\end{abstract}

Alors que le discours de la jeune femme se poursuit, l'image passe au noir après la phrase « Ich hab, glaub ich, wie meistens, irgendwas genäht bei uns in der Küche. » Le bref éclaircissement d'un ou deux photogrammes de l'image avant ce passage au noir, porte à croire qu'il s'agit d'une interruption délibérée de la caméra et non d'un décrochage de fin de bobine. Or cette interruption intervient au moment précis où la jeune femme entame la relation du dernier adieu de Meins, présenté déjà comme un mort vivant, qu'on ne « reverrait que dans les journaux », un personnage qui quitte définitivement le récit oral de ses anciens compagnons pour devenir une figure médiatique. Considérée de la sorte, l'interruption de l'image quelques secondes avant la fin du film s'apparente à un geste méta-discursif. Il indique que les récits qui ont composé le film se posent bel et bien en amont de la carrière terroriste de Meins, en amont de la représentation médiatique de cette carrière, de la mise en scène lors de son arrestation, en amont de son existence en tant qu'image publique $^{17}$.

16 Dans cet extrait des dialogues de Es stirbt allerdings ein Jeder, il s'agit de Ulrike Edschmidt, femme du condisciple de $\mathrm{H}$. Meins et de Gerd Conradt à la DFFB, le réalisateur Enzio Edschmidt.

17 Rappelons par ailleurs que le début du film, avant la première séquence d'interview, nous présente d'abord une série de portraits et d'autoportraits de Meins qui couvrent la période allant de sa naissance à ses études à la DFFB. Ensuite, le fílm se compose exclusivement d'entretiens dont seule une voix sans image survit en fin de film. Cette répartition du matériau constitutif du film indique done, tel est en tout cas notre interprétation, que le film de Renate Sami tente de dresser le portrait d'un homme au moment où il passe à la lutte armée. Ce moment n'est ni celui de ses études ni celui qui suit sa dernière arrestation. Elle correspond à une période sans images. Après avoir payé son dû à l'obligation de l'image, Renate Sami s'en détourne donc définitivement pour aller chercher une vérité au sujet de Meins, non pas dans des synthèses photographiques de sa vie passée, mais dans les souvenirs tout à fait subjectifs de quelques-uns de ses camarades. Dans le cas de Es stirbt allerdings ein Jeder, l'entretien est donc, non pas une conséquence inévitable de l'idéal de contre-information. Il résulte au contraire d'un refus conscient du « tout image (d'archives) » et de la violence sémantique liée à 1'histoire particulière de Holger Meins (exposition télévisuelle de son corps lors de sa dernière arrestation, utilisation d'une photographie médico-légale de sa dépouille décharnée après son décès, etc.). 
Es stirbt allerdings ein Jeder fonde donc son enquête critique et son historiographie du parcours de Meins sur les positions et les récits tout à fait subjectifs de la réalisatrice, rejointe par d'autres auteurs. En somme, comme dans les premiers films réflexifs de Farocki et Sander, 1'auteur est ici un élément indispensable à la critique sociale, politique et réflexive, représentant a contrario un personnage qui reste absent tout au long du film, et suscitant la parole de ceux qui, malgré leur abandon de l'action directe avaient peur de parler $^{18}$.

En raison de son parcours préalable d'activiste, Renate Sami n'est pas tenue de se cacher dans son propre film. Mais au même moment, il en va tout autrement pour une nouvelle génération de réalisateurs engagés qui s'intéressent à leur tour à l'actualité de la lutte armée en Allemagne.

\section{Auteur anonyme et retour de l'auteur}

Dans la plupart des grandes villes de RFA, des groupes dédiés au très jeune médium vidéographique voient le jour dès le début des années soixantedix. Considéré par les militants comme l'outil qui réconcilie action directe et réalisation audiovisuelle, le Porta-pack de Sony, plus léger et surtout moins cher que la caméra pellicule, semble alors répondre parfaitement à l'idée d'un espace public oppositionnel, théorisé abondamment par Oskar Negt et Alexander Kluge ${ }^{9}$, ainsi qu'à la croyance en une réversibilité et un partage total des moyens de communication imaginés par Hans Magnus Enzensberger notamment ${ }^{20}$. Selon les nouveaux vidéastes engagés, la première tâche de l'activiste vidéo consiste à proposer une information alternative qui doit mobiliser les récepteurs devenant producteurs à leur tour, et oter de la sorte la chape de plomb qui recouvre l'information télévisée. $\grave{A}$ cet égard, le projet de l'activisme vidéo reprend à son compte l'idée d'un partage de l'outil audiovisuel et de la suppression subséquente de l'auteur individuel qui avait émergé quelques années auparavant, notamment sur les bancs de la DFFB ${ }^{21}$.

Le dispositif vidéographique doit donc permettre de réaliser l'espace public oppositionnel, Gegenöffentlichkeit, un préliminaire nécessaire à la proletarische Öffentlichkeit, l'espace public prolétarien, sorte de pendant à

18 BEHREND, SAMI, « Holger Meins », p. 19.

19 Oskar NEGT, Alexander KLUGE, Öffentlichkeit und Erfahrung. Zur Organisationsanalyse bürgerlicher und proletarischer Öffentlichkeit, Frankfurt, Suhrkamp Verlag, 1972.

20 Hans Magnus ENZENSBERGER, « Baukasten zu einer Theorie der Medien », Kursbuch, n०20, 1970, p. 159-186.

21 «Projektgruppe anstelle Autor/Regisseur und anstatt anonymer Konsumentenschicht eine Zielgruppe (wobei die Filmprojektgruppe sich aus den politisch Fortschrittlichen der Zielgruppe zusammensetzen soll); tendenzielle Aufhebung der Trennung in Produzent und Konsument [...]; Entmystifizierung der Filmherstellung (jeder könnte Filme machen) [...]. » Günter-Peter STRASCHEK: "Gegen Moralismus, für Konsum », Film, mars 1969, p. 1 et p. 6, cité dans: Hartmut HORST, Wolfgang LOHDING, Operatives Video, Berlin, Medienoperative Berlin e.V., 1977, p. 8. 
l'espace public bourgeois ${ }^{22}$. En raison de la nature notamment médiatique de cet hypothétique espace public d'opposition, la vidéo apparait donc, après l'essoufflement financier et politique d'un partage de l'outil cinématographique à la fin des années soixante, comme un outil taillé sur mesure pour la réalisation de cette sphère publique oppositionnelle.

La plupart des vidéos qui entendent contester le discours médiatique dominant, sont composées d'entretiens et sont le fruit d'un travail collectif. La signature collective ou anonyme est bien évidemment conditionnée par le désir d'un partage de l'outil médiatique par le plus grand nombre ${ }^{23}$. Mais elle apparaît aussi comme un premier bouclier, voire une première riposte à l'individualisation des conflits et des poursuites imaginée par la police et les médias conservateurs et atlantistes. L'entretien, essentiellement avec des proches et des sympathisants des terroristes, est, quant à lui, le seul accès possible à un univers et à un ensemble d'événements inaccessibles.

Cela étant, la lecture parfois un peu rapide des travaux de Negt, de Kluge, ou encore d'Enzensberger, a provoqué une certaine confusion entre espace public oppositionnel et contre-information dans l'esprit des vidéastes engagés ${ }^{24}$. Pour Negt notamment, la pratique médiatique qui prépare l'émergence d'une Gegenöffentlichkeit n'est pas nécessairement contreinformative. Il importe beaucoup plus qu'elle soit d'abord le fait d'une mobilisation, le cas échéant par un auteur extérieur, d'un groupe prolétaire. Ce dernier doit s'emparer ou bénéficier du relais de l'outil audiovisuel. Il peut

22 Si les médias officiels sont en effet des outils pour l'espace public bourgeois et produisent progressivement un écart entre réalité et représentation, l'espace public oppositionnel ne doit pas se satisfaire d'une critique, aussi pertinente soit-elle, de cet outil. Il doit se doter lui aussi d'outils similaires qui élaboreront un « contre-produit » (et non pas une simple contreinformation) opposé au discours déréalisant des médias : «Le genre d'espace public oppositionnel, qui se fonde sur des discours et des idées dont le leitmotiv est la raison, n'est pas en état de déployer des armes tranchantes contre un système se composant de l'illusion, de la publicité et de la puissance publique. Dans une telle situation, les ressorts, que l'espace public bourgeois et classique était en mesure d'activer à l'égard des rapports de violence publics, deviennent de plus en plus désuets. Face à la production d'une publicité sociale illusoire, il s'agit de produire les anticorps d'un espace public prolétarien : idée contre idée, produit social contre produit social, situation productive contre situation productive. " Alexander KLUGE, Oskar NEGT, «Espace public et expérience », in Oskar NEGT, L'espace public oppositionnel, trad. de l'allemand par Alexander NEUMANN, Paris, Payot, 2007, p. 123.

23 Si Enzensberger est le premier penseur allemand du partage de l'outil vidéo, il rappelle aussi dès 1970 le rôle essentiel d'un auteur lors de la première phase de ce partage: « Für den ,Künstler' von ehedem, nennen wir ihn lieber den Autor, folgt aus diesen Überlegungen, daß er sein Ziel darin sehen muß, sich selber als Spezialisten überflüssig zu machen, etwa so, wie der Alphabetisateur seine Aufgabe erst dann erfüllt hat, wenn er nicht mehr benötigt wird. [...] Die taktischen Widersprüche, in die er sich dabei verwickeln muß, lassen sich weder leugnen noch beliebig überspielen. Strategisch aber ist seine Rolle klar. Der Autor hat als Agent der Massen zu arbeiten. Gänzlich verschwinden kann er erst dann in ihnen, wenn sie selbst zu Autoren, den Autoren der Geschichte geworden sind. » ENZENSBERGER, « Baukasten », p. 185-186.

24 Oskar NEGT, "Gegenöffentlichkeit und Erfahrung », in Rudolf MARESCH (éd.), Medien und Öffentlichkeit. Positionierungen, Symptome, Simulationsbrüche, München, Boer, 1996, p. 34-38. 
alors investir un nouvel espace d'expression, définir une nouvelle tribune sociale et s'opposer à la sphère publique bourgeoise.

Si les vidéos proposent effectivement une voix dissonante dans un paysage audiovisuel marqué au mieux par le silence de la critique, au pire par un ensemble de discours réactionnaires, il faut constater aussi que le rêve de l'alternative vidéo aboutit à un échec partiel. L'autodidactisme tout azimut conduit en effet les nouveaux réalisateurs à ne consacrer que peu, voire pas d'importance, à la forme de leurs films. Seul le message compte. Et la contreinformation devient rapidement l'unique raison d'être, urgente, de ces œuvres non fictionnelles ${ }^{25}$. Ces reportages reproduisent alors les canons et clichés formels des médias dominants, contre lesquels ils entendaient pourtant se dresser. Formellement, bon nombre de ces vidéos d'entretiens ne se distinguent que peu en effet des reportages et talk-shows de la télévision de l'époque. Seul le professionnalisme des moyens utilisés et l'habillage diffèrent légèrement. Compte tenu de la réflexion formelle initiée à la fin des années soixante dans les films de Harun Farocki et Helke Sander par exemple, on assiste donc en quelque sorte à une régression en termes d'invention formelle ${ }^{26}$. Le « contre-cinéma » ou la «contre-télévision » sont devenus simple contre-information. Soucieux de favoriser un retour aux personnes concernées par les sujets des films, les vidéos de la première heure s'embourbent dans un dogmatisme stérile qui les place très vite dans la position de simples vis-à-vis, contre-propagandistes, de la télévision. Dès le milieu des années soixante-dix, le schisme devient alors inévitable, revendiqué d'abord par quelques vidéastes conscients que la contreinformation visée par leur travail ne touche essentiellement qu'un " contrepublic », déjà convaincu, un schisme qui culmine bientôt dans la querelle entre les tenants d'une vidéo axée sur le processus de fabrication (prozessorientiertes Video), à laquelle se rattache ouvertement la Medienoperative Berlin par exemple en 1977, et ceux d'une vidéo axée sur les produits (produktorientiertes Video) ${ }^{27}$.

25 Nous songeons notamment à la vidéo d'entretien réalisée par des vidéastes du Medienpädagogikzentrum Hamburg en 1978, Stammheim und Anderswo, qui ne dépasse pas le carcan de l'interview classique et dont les images ne constituent en définitive qu'une conséquence d'un enregistrement sonore placé au premier plan de la narration.

26 Dans son retour réflexif sur l'histoire encore récente de la vidéo en Allemagne, Margret Köhler avance que le travail médiatique critique se fonde sur l'émancipation du citoyen/réalisateur et sur la (contre-)information inédite. Le recours à de nouvelles formes par contre reste totalement absent de cette compréhension de la pratique vidéographique indépendante : « Jede kritische Medienarbeit impliziert Kritik am Fernsehen und der medial vermittelten Wirklichkeit. Die Produktionen sollten sich durch ihren Inhalt und ihre Entstehungsweise von den traditionellen Fernsehproduktionen unterscheiden [...]. » Margret KÖHLER, "Alternative Videoarbeit - Suche nach neuem Selbstvertändnis? », in Margret KÖHLER (éd.), Alternative Medienarbeit. Videogruppen in der Bundesrepublik, « Schriftenreihe des Instituts Jugend Film Fernsehen » t.3, München, Opladen, Leske Verlag + Budrich, 1980, p. 12.

27 Pour une distinction entre ces deux tendances du mouvement vidéo, voir notamment : HORST, LOHDING : «Operatives Video », plus particulièrement p. 51-57. Concernant le 
Dans ce contexte, plusieurs auteurs proches de la DFFB s'emparent à leur tour de l'outil vidéographique. En 1978, Gerd Conradt réalise avec Petra Goldmann Astrid Proll, ihr Leben in England (Astrid Proll, sa vie en Angleterre) dont le gonflage en $16 \mathrm{~mm}$ sera réalisé dans un studio de la DFFB. Et bien que le générique de début de cette vidéo attribue encore la paternité du documentaire à une dizaine de groupes vidéo ouest-allemands, Gerd Conradt et Petra Goldmann s'y exposent et signent physiquement leur documentaire. Au même moment, Wolfgang Höpfner et Norbert Weyer, étudiants à la DFFB, réalisent Vor 4 Jahren, vor 2 Jahren (Il y a 4 ans, il y a 2 ans, 1977-1979), consacré à Philipp Werner Sauber, abattu par la police à Cologne en mai 1975. Ici aussi, les deux réalisateurs n'hésitent pas à s'exposer en tant qu'auteurs dans le film, sans toutefois que cette position ne les préserve du travers contre-informatif qui "handicape » encore certaines séquences.

Dès 1975, Gerd Conradt et Hartmut Jahn s'attellent à la réalisation d'une vidéo dédiée à Holger Meins et qui aboutit en 1982 au documentaire Über Holger Meins. Unsere Sicht heute (Holger Meins. Notre vision aujourd'hui). En 1978, c'est-à-dire trois années après le début du tournage, une première version inachevée de la vidéo voit le jour. Cette version intitulée Über Holger Meins est présentée cette année-là lors de quelques rares diffusions dans des centres vidéo allemands comme le produit provisoire d'un work in progress ${ }^{28}$. C'est à une séquence de cette version jamais commentée ou analysée, que nous nous intéressons dans la suite de cet article.

\section{Au-delà de la césure temporelle}

Sans épuiser toutes les facettes de cette ouvre singulière qu'est Über Holger Meins, nous aimerions en explorer une séquence qui, en dépit des apparences, entre en résonance directe avec le film de Renate Sami, et qui suggère à son tour que durant la seconde moitié des années soixante-dix, entre militantisme vidéographique direct et reportage mainstream, des auteurs liés à la DFFB explorent une tierce voie dans la représentation du terrorisme d'extrême gauche allemand. À l'instar de Sami, Conradt et Jahn dépassent en effet la critique simple, la contre-information non circonstanciée et la violence sémantique qui caractérise les portraits médiatiques de Meins, pour produire une œuvre réflexive et critique.

constat d'incfficacité politique et d'imperfection tcchnique des vidéos contestataires, voir notamment : Margret KÖHLER, «Dic 'dritte Generation' auf der Suche nach einem Selbstverständnis. Das VIDEO-FORUM in Berlin vom 1. bis 5. Dezember 1978 ", in Medien + Erziehung, n $^{\circ} 1$, 1979, p. 54 ; HORST, LOHDING, «Operatives Vidco », p. 75.

28 Nous tenons à remercicr ici Monsicur Olaf Bcrg du Medienpädagogikzentrum dc Hambourg qui nous a été d'une aide inestimable lors de la recherche quasi alćatoire d'une version inachevée de la vidćo de Conradt et Jahn. 
Dans la séquence qui nous intéresse, nous assistons tout d'abord à une succession au rythme soutenu d'images du leader estudiantin Fritz Teufel sur le banc des accusés dans une salle d'audience, des photographies de titres diffamatoires voire outrageux à l'égard de Teufel et de ses collègues étudiants à la une de journaux à sensation, et des extraits d'actes d'accusation et de protocoles de placement en détention préventive. Après ce montage rapide et non commenté d'images issues probablement de l'œuvre inachevée de Meins, Über Fritz Teufel, on voit un très long extrait du film de Harun Farocki, Die Worte des Vorsitzenden (1967 ; caméra : Holger Meins). Cet extrait est suivi immédiatement de plans d'actualités mais aussi d'images d'amateurs, qui évoquent très rapidement les bombardements au Vietnam et la répression policière de manifestations étudiantes ${ }^{29}$. Ensuite, un nouvel extrait de ce qui est probablement un autre film inachevé de Meins, $B Z$ ins Klosett ( $A u x W C$, la Berliner Zeitung), nous confronte à une succession extrêmement rapide de cinq plans brefs (moins d'une seconde chacun), montés en boucle, qui suggèrent que la $B Z$ ne vaut guère mieux qu'un papier toilette. Après un bref passage par un extrait d'un autre film de Harun Farocki, Ihre Zeitungen (1968), le montage revient à des images d'actualité ou des images tournées par des cinéastes amateurs, cette fois lors d'assauts étudiants sur les bâtiments du groupe d'édition Springer à Berlin. Suivent quelques minutes d'une conversation filmée entre Holger Meins et un jeune homme que nous n'avons pas identifié, qui porte sur la conscience du révolutionnaire, après quoi la séquence en arrive enfin à des images originales tournées par Conradt et Jahn. Le père de Holger Meins y commente et condamne la première arrestation arbitraire de son fils par la police allemande et le traitement médiatique de cette arrestation. Un peu plus loin, Conradt interroge encore le père sur son quotidien et sur ses contacts avec une veuve de guerre qui rend régulièrement visite au vieil homme. Ces deux extraits d'entretien avec Wilhelm Meins sont séparés par un fragment d'un reportage traitant du prix exorbitant de l'entretien et de la consommation en carburant des Starfighters, avions de chasse controversés de l'armée de l'air allemande. Enfin, cette séquence très hétéroclite se termine par les images célèbres de la dernière arrestation de Meins : l'évacuation du jeune terroriste, nu, se tordant de douleur et hurlant de rage. Le moment très précis de son cri est répété, au ralenti, trame par trame.

Au-delà de son désordre apparent, cette longue séquence propose quelques rapprochements significatifs essentiels pour notre propos. La juxtaposition d'un ensemble d'images de remploi suggère d'abord que le Vietnam, les rues de Berlin, les tribunaux allemands, le bâtiment du groupe d'édition Springer et la Bundeswehr de la fin des années soixante-dix appartiennent en réalité à un seul et même complexe ou champ de bataille. Au nom d'une même violence, un groupe d'édition pro-atlantiste, la police allemande et l'armée

29 Il pourrait s'agir en l'espèce d'extraits du film de Thomas Giefer et Hans-Rüdiger Minow, étudiants à la DFFB, Der 2. Juni 1967 (1967). 
américaine sont ainsi associés dans la succession très rapide d'images tournées au Vietnam et à Berlin, qui disent littéralement qu'entre le soldat américain et le policier berlinois, il n'y a plus aucune différence ${ }^{30}$. En ce sens les images de l'arrestation de Meins accusent, elles aussi, un seul et grand complexe médiatico-policier dénoncé ici dans ce qu'il aurait de plus inhumain.

Par ailleurs, la séquence que nous venons de décrire succinctement, relève a priori d'une approche documentaire de la figure du personnage absent, opposée en bien des points à la narration proposée par Renate Sami dans Es stirbt allerdings ein Jeder : grâce à un retour en images sur l'histoire d'une génération d'étudiants engagés, le spectateur peut se remémorer un contexte médiatique et cinématographique déterminant pour le parcours politique de Holger Meins. À ce stade du film, le portrait du personnage principal mais absent s'appuie donc sur une rencontre entre les rares traces en images qui nous restent du jeune homme (autoportraits et images tournées par Meins) et les images d'une époque qui a nourri ses choix de vie. Mais la séquence augmente cette approche documentaire d'une dimension réflexive qui met non seulement la pratique cinématographique de Meins à distance mais aussi le retour sur cette pratique par Conradt et Jahn. Car en mêlant littéralement des images tournées par Meins à des plans réalisés pour les besoins du portrait posthume, Über Holger Meins recompose aussi et achève en quelque sorte le grand projet de l'apprenti cinéaste, militant et futur terroriste. Au début de cette séquence, le commentaire en off nous apprend en effet qu'avant de se détourner du cinéma, Meins avait mis en chantier un long film militant composé de plusieurs courts métrages. En recyclant des extraits de ses œuvres inachevées BZ ins Klosett et Über Fritz Teufel, et en leur adjoignant des extraits d'autres films réalisés par les premiers étudiants de la DFFB et des fragments de reportages officiels, Conradt et Jahn se substituent donc au réalisateur absent, Holger Meins, pour achever un grand travail d'assemblage, que Meins n'avait pas pris le temps de terminer, interrompu qu'il fut par son passage à la clandestinité.

Pour évoquer le passé de cinéaste de leur ancien condisciple sur les bancs de la DFFB, Conradt et Jahn clôturent donc à leur façon le projet cinématographique de Meins, entamé quelque dix années plus tôt, en proposant un long montage d'extraits hétérogènes de films et de reportages réalisés entre 1966 (ouverture de la DFFB) et 1972 (arrestation de Holger Meins). Mais réfutant une historiographie de la rétrospective qui trouverait dans les images du futur terroriste les signes avant-coureurs de son action

30 Très en vogue dans les milieux estudiantins de la fin des années soixante, 1'indistinction entre violence policière et violence militaire au nom d'une lutte marxiste sur tous les fronts, se trouve bien évidemment aussi aux origines de la fondation de groupes tels que la Fraction Armée Rouge. On le sait, ces groupes entendaient non seulement combattre l'ennemi américain sur le territoire allemand mais se plaisaient aussi à se considérer comme les légataires allemands de groupes armés luttant pour la révolution anti-impérialiste dans le monde (Asie, Amérique latine, Afrique). 
violente, les deux vidéastes n'hésitent pas à s'approprier et à réarticuler les rushes de Meins par un travail singulier de juxtaposition. Et comme ce travail ne se limite pas aux images d'archives, il suggère dès lors que si les agit-films mais aussi les images d'actualités réalisées entre 1966 et 1972 délimitent ensemble un contexte visuel, le film de Conradt et Jahn fait partie intégrante de ce contexte et donc d'une histoire de l'engagement cinématographique initié dix années plus tôt. Entourés d'images qui renvoient le spectateur à une époque révolue, les plans du père de Meins par exemple s'intègrent à un retour en images sur l'histoire des images. En ce sens, la cohabitation d'archives réemployées et d'images plus récentes réalisées par Conradt et Jahn, démontre que Über Holger Meins s'inscrit véritablement dans une démarche que la plupart de leurs camarades d'école de la DFFB ont abandonnée depuis longtemps. En d'autres termes, le remontage auquel s'essayent Conradt et Jahn confère à leur vidéo une dimension résolument autoréflexive : le montage ne se borne pas seulement à évoquer l'époque révolue d'un usage militant et agitateur du cinéma pour dessiner le portrait d'un personnage définitivement absent. Le montage se regarde aussi luimême.

$\grave{A}$ la fin, le documentaire se retourne encore, de façon tout à fait explicite cette fois, sur lui-même, lorsqu'un jeune homme non identifié rend visite au père de Holger Meins pour lui faire part de son admiration. Ici, l'évocation du courage d'un père resté fidèle à son fils malgré les pressions politiques et sociales, permet de dépasser l'écart générationnel qui persiste bien évidemment entre Conradt et le père Meins :

[...] als ich den Film gesehen habe, also diese Skizze jetzt, diesen Entwurf, für mich war das Erstaunlichste daran, ich sagte es vorhin schon, ihre Solidarität, nicht? Zum Beispiel, meine Eltern hätten das nicht geschafft. Ich sag das so, nicht? Und viele Leute würden das nicht schaffen, diesen Konflikt zwischen den Kindern und der Gesellschaft, [...] mit den Kindern solidarisch sein, nicht. Sie hätten sich für die Anerkennung und den Frieden mit ihrer Umgebung gegen das Kind oder gegen die Kinder entschieden. [...] Und ich glaube schon, dass das, also dass der Zwang und die Bedrohung von außen sehr, sehr groß für sie gewesen sein muss ${ }^{31}$.

Placé à la fin du documentaire, ce discours suggère une transgression de la polarisation générationnelle et temporelle, opérée par le père grâce au documentaire. Il met en évidence un père de terroriste, seul, rêvant certes à une autre trajectoire pour son fils décédé, mais qui défend néanmoins la mémoire de son enfant en attaquant frontalement l'État et les médias. Forte d'autres parallélismes entre Gerd Conradt et Wilhelm Meins notamment, et auxquels nous ne nous attardons pas dans le présent texte, la parole du jeune homme fait aussi écho à la résistance au temps qui passe. Là où dans le film de Renate Sami, Hartmut Bitomsky abordait ses illusions perdues, Conradt et Jahn cherchent à prolonger un engagement cinématographique en augmentant

31 Extrait des dialogues de Über Holger Meins. 
leur regard sur les images du passé par un éclairage réciproque des images du présent : leur " vue aujourd'hui », selon le titre du film quatre ans plus tard. Si Über Holger Meins bat donc ici en brèche toute la composante générationnelle des conflits et débats politiques des années soixante et soixante-dix et dépasse ainsi la polarisation d'un conflit dont les camps sont toujours prétablis, il propose aussi une échappatoire réflexive à la fracture temporelle qui a eu raison de l'engagement des camarades de Meins cinéaste.

\section{Coda}

$\mathrm{Au}$ terme de cette réflexion parcellaire au sujet de deux documentaires réalisés durant "les années de plomb » allemandes, nous avons essayé de remédier à une cécité partielle qui affecte encore bon nombre d'histoires du cinéma allemand. Ce texte ne constitue bien évidemment qu'un premier pas dans ce sens. Mais il veut ouvrir la recherche à tous ces films et toutes ces vidéos qui démontrent qu'à rebours d'une lecture conventionnelle des années soixante et de leurs suites, la création audiovisuelle n'a eu de cesse de repenser les liens entre engagement politique sur fond de lutte armée et innovation formelle.

Au cours des recherches qui ont précédé notre réflexion, nous avons visité d'anciens centres vidéo et diverses structures, parfois moribondes, qui tentent aujourd'hui encore, dans la limite de leurs moyens financiers, de rendre les œuvres accessibles au grand public comme au chercheur. Souvent, nous avons dû rebrousser chemin, arrêté par l'inaccessibilité matérielle de certaines bandes vidéo référencées par les centres et dont les titres ne laissaient aucun doute quant à leur lien avec notre sujet. Affectés par le temps, d'innombrables documentaires sont aujourd'hui irregardables. Dans certains cas, les bandes se sont littéralement agglutinées, collées, et le passage, même au ralenti, par un lecteur, provoquerait immédiatement une déchirure du support synthétique devenu extrêmement fragile. La restauration de ces bandes exige des moyens qui ne peuvent être dégagés par les dépositaires de ces documents. Bien évidemment, il faut trouver aujourd'hui les moyens nécessaires à ce travail d'orfèvre, car la disparition progressive de ces documents précieux risque, dans un délai très court, de priver les archivistes et historiens d'un matériau historiographique majeur. Mais s'engager dans la restauration de ces bandes revient aussi à accomplir un acte de résistance. Résistance à l'histoire instituée qui considère trop souvent l'engagement politique d'un "contrecinéma » comme un mort-né de la fin des années soixante. Trouver, référencer et rendre accessible ce « contre-cinéma », c'est lui rendre sa place originelle : celle d'un outil qui a écrit une part de «contre-histoire ». 